\title{
Late Cretaceous to Paleogene exhumation in Central Europe - localized inversion vs. large-scale domal uplift
}

Hilmar von Eynatten ${ }^{1}$, Jonas Kley ${ }^{2}$, István Dunkl ${ }^{1}$, Veit-Enno Hoffmann ${ }^{1}$, Annemarie Simon ${ }^{1}$

${ }^{1}$ University of Göttingen, Geoscience Center, Department of Sedimentology and Environmental Geology, Goldschmidtstrasse 3, 37077 Göttingen, Germany

${ }^{2}$ University of Göttingen, Geoscience Center, Department of Structural Geology and Geodynamics,

Goldschmidtstrasse 3, 37077 Göttingen, Germany

Correspondence to: Hilmar von Eynatten (heynatt@gwdg.de)

\section{Supplement:}

S1, Table: List of the thermochronologial samples including locality, stratigraphy and petrography.

S2, Figure: Map illustrating sample locations, based on: Geowissenschaftliche Karte der Bundesrepublik Deutschland 1: 2000 000, Bundesanstalt für Geowissenschaften und Rohstoffe, Hannover (2004).

S3, Table: Apatite fission track data $(\mathrm{n}=110)$

S4, Figure: Binned diagrams of horizontal confined fission track lengths measured in the apatite samples.

S5, Table: Apatite (U-Th)/He data $(n=37)$. 


\begin{tabular}{|c|c|c|}
\hline Sample & Region $^{a}$ & Locality \\
\hline V-7 & & Ellrich \\
\hline V-19 & W-BM & Forschengereuth \\
\hline V-24 & TF & Mosbach \\
\hline V-25 & TF & Mosbach \\
\hline V-27 & TF & Fuchsberg/Seebach \\
\hline V-28 & TF & Wartburg \\
\hline$V-29$ & TF & Bromacker \\
\hline V-30 & TF & Bromacker \\
\hline V-31 & FP & Fambach \\
\hline V-32 & TF & Rennsteig \\
\hline V-59 & WFW & Dorndorf, Rhön \\
\hline V-60 & FP & Hindfeld \\
\hline V-61 & TB & Arnstadt \\
\hline V-63 & TB & Rockensußra-2, core $442 \mathrm{~m}$ \\
\hline V-64 & TB & Rockensußra-2, core $446 \mathrm{~m}$ \\
\hline V-80 & W-BM & Gölschtal \\
\hline V-85 & W-BM & Schafberg / Weida \\
\hline V-86 & W-BM & Gera \\
\hline V-94 & TB & Holzhausen \\
\hline V-95 & & Heseberg \\
\hline V-96 & E-RM & Wilhemshütte \\
\hline V-97 & E-RM & Somplar \\
\hline V-99 & E-RM & Somplar,Meiserhof \\
\hline V-101 & E-RM & Neu-Ludwigsdorf \\
\hline V-103 & E-RM & Frankenberg \\
\hline V-108 & E-RM & Dainrode \\
\hline V-109 & E-RM & Brinkhausen \\
\hline V-110 & E-RM & Frankenau \\
\hline $\mathrm{V}-112$ & E-RM & Somplar-Anteholz \\
\hline$V-113$ & E-RM & Wilhelmshütte \\
\hline V-115 & E-RM & Würzberg \\
\hline V-121 & WFW & Schedebachtal \\
\hline $\mathrm{V}-127$ & WFW & Reinhausen \\
\hline$V-128$ & & Aschersleben \\
\hline V-130 & & Mansfeld \\
\hline V-131 & & Annaroda \\
\hline$V-132$ & & Paßbruch \\
\hline V-133 & & Wettelrode \\
\hline$V-135$ & & Anaroda \\
\hline$V-136$ & & Brücken \\
\hline$V-137$ & & Feld Sittendorf \\
\hline$V-138$ & & Hettstedt \\
\hline V-139 & WFW & bei Dasandstoneel \\
\hline V-141 & WFW & Amelungsborn \\
\hline$V-143$ & WFW & Offensen \\
\hline V-144 & WFW & Mariaspring \\
\hline$V-145$ & WFW & Die Clus \\
\hline V-146 & WFW & Stadtoldendorf \\
\hline V-147 & WFW & Dasandstoneel \\
\hline V-148 & WFW & Löwenhagen \\
\hline V-151 & $\mathrm{FP}$ & Frickenhausen \\
\hline V-154 & FP & Schleusingen \\
\hline V-155 & & Langenbach \\
\hline V-156 & WFW & Hof Guttels \\
\hline V-157 & WFW & Blankenbach \\
\hline V-158 & WFW & Dens \\
\hline V-159 & WFW & Unterellen \\
\hline$V-160$ & WFW & Nesandstoneelröden \\
\hline V-161 & WFW & Melsungen \\
\hline$V-162$ & WFW & Kloster Cornberg \\
\hline$V-163$ & WFW & Weißenborn \\
\hline V-165 & W-BM & Jena-Göschwitz \\
\hline V-166 & W-BM & Kraftsdorf \\
\hline V-167 & W-BM & Altendorf \\
\hline V-168 & W-BM & Orlamünde \\
\hline V-169 & W-BM & Weißbach \\
\hline V-171 & W-BM & Remschütz \\
\hline V-172 & W-BM & Teichel \\
\hline V-173 & W-BM & Großeutersdorf \\
\hline V-183 & TB & Wachsenburg \\
\hline V-191 & E-RM & Fritzlar \\
\hline V-192 & E-RM & Rösenbeck \\
\hline V-194 & E-RM & Lenne, Wesenberg \\
\hline V-197 & E-RM & Wallenstein \\
\hline$V-201$ & & Badra \\
\hline$V-203$ & & Artern \\
\hline$V-204$ & & Kachstedt \\
\hline
\end{tabular}

Stratigraphy ${ }^{b}$

Permian (Upper Rotliegend)

Lower Triassic (su/sm)

Lower Triassic (su)

Lower Triassic (sm)

Lower Triassic (su)

Permian (Upper Rotliegend)

Permian (Upper Rotliegend)

Permian (Upper Rotliegend)

Lower Triassic (su)

Permian (Rotliegend)

Lower Triassic (su)

Upper Triassic $(\mathrm{km})$

Upper Triassic $(\mathrm{km})$

Lower Triassic (sm)

Lower Triassic (sm)

Variscan basement

Lower Carboniferous

Lower Carboniferous

Upper Triassic (ku)

Lower Triassic (sm)

Lower Carboniferous

Lower Carboniferous

Lower Carboniferous

Lower Carboniferous

Lower Carboniferous

Lower Carboniferous

Lower Carboniferous

Lower Carboniferous

Lower Carboniferous

Lower Carboniferous

Lower Triassic (sm)

Lower Triassic (sm)

Lower Triassic (sm)

Lower Triassic (sm)

Permian (Upper Rotliegend)

Permian (Upper Rotliegend)

Carboniferous

Permian (Upper Rotliegend)

Lower Triassic (su)

Lower Triassic (sm)

Lower Triassic (sm)

Permian (Upper Rotliegend)

Lower Triassic (sm)

Lower Triassic (sm)

Lower Triassic (sm)

Lower Triassic (sm)

Lower Triassic (sm)

Lower Triassic (sm)

Lower Triassic (sm)

Lower Triassic (sm)

Lower Triassic (sm)

Lower Triassic (su)

Permian (Lower Rotliegend)

Lower Triassic (sm)

Lower Triassic (sm)

Lower Triassic (sm)

Lower Triassic (sm)

Lower Triassic (sm)

Lower Triassic (sm)

Permian (Upper Rotliegend)

Lower Triassic (sm)

Lower Triassic (sm)

Lower Triassic (sm)

Lower Triassic (sm)

Lower Triassic (sm)

Lower Triassic (sm)

Lower Triassic (sm)

Lower Triassic (sm)

Lower Triassic (sm)

Upper Triassic (ku)

Lower Triassic (sm)

Middle Devonian

Middle Devonian

Middle Devonian

Lower Triassic (su)

Lower Triassic (su)

Lower Triassic (su)
Petrography

51,585222

Lat $\left[^{\circ}\right]$

Elev. [m]

sandstone $\quad 50,392355 \quad 11,137033 \quad 565$

sandstone $\quad 50,929958 \quad 10,354174 \quad 334$

sandstone $\quad 50,929786 \quad 10,355083 \quad 334$

sandstone $\quad 50,920146 \quad 10,417938 \quad 397$

granite-cobble $\quad 50,967845 \quad 10,304182 \quad 347$

sandstone $\quad 50,810852-10,621213$

sandstone $\quad 50,810852 \quad 10,621213$

sandstone $\quad 50,738028 \quad 10,376858 \quad 283$

rhyolite $\quad 50,834311 \quad 10,532340 \quad 536$

sandstone $\quad 50,834958 \quad 10,084072 \quad 245$

sandstone $\quad 50,367103 \quad 10,566521 \quad 302$

sandstone $\quad 50,837842 \quad 10,944055 \quad 272$

sandstone $\quad 51,267691 \quad 10,700165 \quad-170^{\mathrm{C}}$

sandstone $\quad 51,267691 \quad 10,700165 \quad-174^{\mathrm{C}}$

granite $\quad 50,622916 \quad 12,241825 \quad 317$

conglomerate $\quad 50,789141 \quad 12,082128 \quad 252$

conglomerate $\quad 50,834784 \quad 12,086967 \quad 225$

$\begin{array}{llll}\text { sandstone } & 50,845825 & 10,880198 & 369\end{array}$

sandstone $\quad 52,083635 \quad 10,871253 \quad 186$

wacke $\quad 51,061939 \quad 8,623232 \quad 485$

wacke $\quad 51,065802 \quad 8,724715 \quad 370$

wacke $\quad 51,059129 \quad 8,739578 \quad 316$

wacke $\quad 51,070018 \quad 8,618118 \quad 515$

wacke $\quad 51,062918 \quad 8,792114$

wacke

wacke $\quad 51,173626 \quad 8,999682$

wacke $\quad 51,077999 \quad 8,921126$

wacke

wacke $\quad 51,062044 \quad 8,624253$

sandstone $\quad 50,974371 \quad 8,754202$

sandstone $\quad 51,446737 \quad 9,670660$

sandstone $\quad 51,467934 \quad 9,983361$

sandstone $\quad 51,781411 \quad 11,552616$

$51,605972-11,472827$

$51,544210 \quad 11,395198$

$51,548518 \quad 11,136968$

$51,521606 \quad 11,260087$

$51,514886 \quad 11,374665$

$51,435792 \quad 11,183399$

$51,449664 \quad 11,104679$

$51,644602 \quad 11,474151$

$51,743493 \quad 9,651987$

$51,918430 \quad 9,620183$

$51,558938 \quad 9,718127$

$51,599214 \quad 9,951020$

$51,815923 \quad 9,920936$

$51,885350 \quad 9,620466$

$51,799856 \quad 9,669938$

$51,530527 \quad 9,671852$

50,417445

50,504771

50,506626

51,012059

51,002256

51,017994

50,981664

51,038424

51,118450

51,042973

51,077552

50,874605

50,878126

50,833547

50,775577

50,715618

50,689264

50,780742

50,791739

50,856987

51,130434

51,413735

51,165575

51,326595

51,403515

51,380839

51,390663

10,230381

10,766366

10,847554

9,703412

10,002735

9,900992

10,161005

10,093233

9,538753

9,864981

9,964347

11,596590

11,930859

11,587526

11,538074

11,454526

11,378823

11,313983

11,567417

10,875799

9,217180

8,716847

8,397016

8,172341

10,971743

sandstone

11,282435

11,265663 


\begin{tabular}{|c|c|c|c|c|c|c|c|}
\hline$V-206$ & E-RM & Wüstung Lanzberg & Lower Triassic (sm) & sandstone & 51,367758 & 9,159326 & 277 \\
\hline V-207 & WFW & Heiligenstadt & Lower Triassic (sm) & sandstone & 51,396309 & 10,128237 & 328 \\
\hline$V-216$ & WFW & Eichenberg & Upper Triassic (ko) & quartzarenite & 51,381876 & 9,933640 & 212 \\
\hline$V-217$ & W-BM & Langenorla & Lower Triassic (sm) & sandstone & 50,744255 & 11,571874 & 195 \\
\hline$V-218$ & TB & Erfurt-1, core $305 \mathrm{~m}$ & Lower Triassic (sm) & sandstone & 50,907677 & 11,192894 & $158^{\mathrm{c}}$ \\
\hline$V-219$ & $\mathrm{~TB}$ & Tennstedt- 1 core $447 \mathrm{~m}$ & Lower Triassic (sm) & sandstone & 51,155639 & 10,815234 & $-271^{c}$ \\
\hline$V-220$ & W-BM & Schmücke-Tunnel & Lower Triassic (sm) & sandstone & 51,279870 & 11,220210 & 213 \\
\hline$V-222$ & WFW & Arenshausen & Lower Triassic (sm) & sandstone & 51,377112 & 9,975211 & 206 \\
\hline V-224 & E-RM & Hofgut Teiske & Lower Carboniferous & wacke & 51,319086 & 8,761191 & 452 \\
\hline V-225 & W-BM & Finnetunnel & Lower Triassic (su/sm) & sandstone & 51,163570 & 11,484093 & 230 \\
\hline$V-226$ & E-RM & Schweinsbühl & Lower Carboniferous & wacke & 51,314457 & 8,746747 & 531 \\
\hline$V-227$ & E-RM & Diemelstadt-Rohde & Lower Triassic (sm) & sandstone & 51,478454 & 9,019170 & 265 \\
\hline$V-228$ & W-BM & Niederpölniz & Lower Carboniferous & wacke & 50,754319 & 11,976597 & 346 \\
\hline$V-230$ & $\mathrm{FP}$ & Hindfeld & Upper Triassic (km) & sandstone & 50,367825 & 10,564119 & 306 \\
\hline$V-231$ & W-BM & Großmonra & Upper Triassic $(\mathrm{km})$ & sandstone & 51,212569 & 11,295544 & 184 \\
\hline V-233 & & Sonnenstein & Lower Triassic (sm) & sandstone & 51,511301 & 10,391112 & 355 \\
\hline$V-234$ & WFW & A4, Moseberg & Lower Jurassic & sandstone & 51,009268 & 10,276924 & 234 \\
\hline$V-235$ & WFW & Breitenbach & Lower Triassic (sm) & sandstone & 51,401930 & 10,311604 & 405 \\
\hline$V-238$ & E-RM & Lölbach & Lower Carboniferous & wacke & 51,069656 & 8,992817 & 506 \\
\hline V-239 & E-RM & Grüsandstoneen & Lower Triassic (sm) & sandstone & 50,991716 & 8,966718 & 266 \\
\hline$V-242$ & E-RM & Dringe & Lower Triassic (sm) & sandstone & 51,220948 & 9,068341 & 362 \\
\hline$V-245$ & E-RM & Vasbeck & Lower Triassic (sm) & sandstone & 51,382295 & 8,875809 & 383 \\
\hline V-252 & E-RM & Stbr. Hahneberg & Devonian & sandstone & 51,102361 & 9,067175 & 471 \\
\hline$V-254$ & E-RM & Gemünden & Lower Carboniferous & quartzite & 50,974045 & 9,020218 & 365 \\
\hline$V-256$ & E-RM & Burg Waldeck & Lower Carboniferous & wacke & 51,200973 & 9,060579 & 264 \\
\hline V-257 & E-RM & Sachsenhausen & Lower Triassic (sm) & sandstone & 51,249618 & 8,996912 & 393 \\
\hline V-258 & E-RM & Frankenerg & Upper Permian (Zechstein) & siltstone & 51,070559 & 8,768689 & 338 \\
\hline$V-259$ & E-RM & Hundshausen & Carboniferous & wacke & 50,981928 & 9,135671 & 264 \\
\hline$V-265$ & WFW & Feldhain, Meißner & Lower Triassic (sm) & sandstone & 51,247840 & 9,892266 & 360 \\
\hline$V-266$ & WFW & Schafshof/Hölltal & Upper Devonian & wacke & 51,229490 & 9,944367 & 237 \\
\hline$V-272$ & $\mathrm{FP}$ & Bad Kissingen & Lower Triassic (sm) & sandstone & 50,199386 & 10,050547 & 293 \\
\hline$V-273$ & FP & Popenhausen & Upper Triassic (ku) & siltstone & 50,098917 & 10,157208 & 300 \\
\hline$V-274$ & WFW & Krukenburg & Lower Triassic (sm) & sandstone & 51,631479 & 9,458577 & 146 \\
\hline
\end{tabular}

a: according to Figures 3 and 4: TB - Thuringian Basin, TF - Thuringian Forest, WFW - Werra-Fulda-Weser region, E-RM: Eastern Rhenish Massif, FP: Franconian Platform, W-BM: Western Bohemian Massif.

b. su, sm, and so are abbreviations for Lower, Middle and Upper Buntsandstein, respectively. The same applies to ku, km, and ko for Keuper subunits.

c: For drillcore samples the depth below the surface is given under locality; the indicated elevation is always relative to sea level. 


\begin{tabular}{|c|c|c|c|c|c|c|c|c|c|c|c|c|c|c|c|c|}
\hline Sample & $\mathbf{n}$ & $\operatorname{RhoS}^{\mathrm{a}}$ & $(\mathrm{Ns})^{\mathrm{b}}$ & Rhol & (Ni) & RhoD $^{c}$ & (Nd) & $P(\%)^{d}$ & Disp. $^{e}$ & Central Age & $\pm 1 s$ & Uran. $^{f}$ & $\mathrm{MTL}^{\mathrm{g}}$ & $\pm 1 \mathrm{~s}$ & $\mathbf{n}$ & Dpar \\
\hline V-7 & 25 & 16,8 & 916 & 19,4 & 1061 & 7,12 & 5610 & 1 & 0,19 & 101,5 & 6,4 & 33 & 12,3 & 2,3 & 58 & 2,21 \\
\hline V-19 & 20 & 19,7 & 1146 & 17,4 & 1011 & 7,06 & 5252 & 80 & 0,00 & 128 & 6,1 & 30 & 12,0 & 2,2 & 68 & 1,87 \\
\hline V-24 & 21 & 27,7 & 1257 & 37,8 & 1714 & 6,88 & 5252 & 76 & 0,00 & 81 & 3,4 & 63 & 13,7 & 1,2 & 60 & 2,14 \\
\hline V-25 & 23 & 15,5 & 701 & 16,5 & 745 & 5,54 & 5232 & 74 & 0,01 & 83,6 & 4,7 & 34 & 13,2 & 1,8 & 59 & 2,18 \\
\hline V-27 & 21 & 17,3 & 1275 & 23,8 & 1750 & 6,85 & 5252 & 98 & 0,00 & 80,1 & 3,4 & 42 & 13,6 & 1,3 & 61 & 2,11 \\
\hline V-28 & 24 & 11,3 & 751 & 17,2 & 1147 & 7,00 & 5252 & 96 & 0,00 & 73,6 & 3,8 & 29 & 13,1 & 1,8 & 60 & 1,98 \\
\hline V-29 & 23 & 22,5 & 1575 & 35,8 & 2508 & 7,01 & 5252 & 87 & 0,00 & 70,8 & 2,7 & 58 & 13,8 & 1,2 & 50 & 2,28 \\
\hline V-30 & 24 & 14,2 & 631 & 18,9 & 839 & 5,57 & 5232 & 91 & 0,00 & 67,4 & 3,8 & 41 & 13,6 & 1,3 & 61 & 2,23 \\
\hline V-31 & 25 & 16,9 & 1396 & 27,0 & 2228 & 6,99 & 5252 & 95 & 0,00 & 70,4 & 2,8 & 45 & 13,6 & 1,2 & 61 & 2,06 \\
\hline V-32 & 14 & 6,1 & 203 & 9,3 & 307 & 6,90 & 5252 & 97 & 0,00 & 73,3 & 6,8 & 16 & & & & 2,12 \\
\hline V-59 & 24 & 15,6 & 883 & 20,8 & 1180 & 5,62 & 5232 & 100 & 0,00 & 67,7 & 3,3 & 43 & 13,4 & 1,3 & 58 & 2,14 \\
\hline V-60 & 22 & 11,6 & 602 & 15,8 & 820 & 5,59 & 5232 & 100 & 0,00 & 66 & 3,8 & 33 & 12,3 & 1,7 & 60 & 2 \\
\hline V-61 & 23 & 18,7 & 1015 & 24,8 & 1347 & 6,36 & 6013 & 100 & 0,00 & 76,9 & 3,5 & 45 & 12,7 & 1,6 & 60 & 2,2 \\
\hline V-63 & 24 & 24,0 & 1266 & 35,7 & 1885 & 6,92 & 5252 & 95 & 0,00 & 74,6 & 3,1 & 60 & 12,7 & 2,2 & 58 & 2,16 \\
\hline V-64 & 22 & 24,8 & 1604 & 35,8 & 2313 & 6,95 & 5252 & 88 & 0,00 & 77,4 & 3 & 62 & 11,7 & 1,8 & 45 & 2,08 \\
\hline V-80 & 21 & 47,8 & 1606 & 38,0 & 1279 & 6,62 & 6378 & 79 & 0,00 & 132,9 & 5,6 & 69 & 12,1 & 1,8 & 61 & 1,7 \\
\hline V-85 & 22 & 16,1 & 944 & 11,9 & 694 & 6,96 & 5252 & 48 & 0,04 & 151,3 & 8,3 & 21 & 13,3 & 1,4 & 60 & 2,25 \\
\hline V-86 & 17 & 16,4 & 730 & 20,8 & 924 & 6,89 & 5252 & 51 & 0,01 & 87,4 & 4,7 & 34 & 12,7 & 1,8 & 64 & 2,17 \\
\hline V-94 & 25 & 14,8 & 812 & 22,8 & 1249 & 6,57 & 6378 & 98 & 0,00 & 68,7 & 3,4 & 44 & 13,9 & 1,2 & 59 & 2,62 \\
\hline V-95 & 23 & 21,3 & 635 & 25,9 & 770 & 6,18 & 6013 & 100 & 0,00 & 81,9 & 4,7 & 51 & 12,7 & 2,0 & 60 & 2,5 \\
\hline V-96 & 20 & 13,1 & 665 & 14,7 & 747 & 6,66 & 6378 & 98 & 0,00 & 95,1 & 5,4 & 27 & 13,3 & 1,6 & 60 & 2,24 \\
\hline V-97 & 24 & 14,9 & 882 & 14,2 & 842 & 6,64 & 6378 & 92 & 0,00 & 111,5 & 5,8 & 26 & 13,5 & 1,4 & 60 & 2,42 \\
\hline V-99 & 25 & 12,9 & 795 & 13,6 & 841 & 6,51 & 6378 & 100 & 0,00 & 98,7 & 5,2 & 25 & 13,4 & 1,7 & 60 & 3,03 \\
\hline V-101 & 25 & 9,6 & 407 & 13,4 & 570 & 6,39 & 6013 & 100 & 0,00 & 73,3 & 5 & 25 & 12,9 & 1,7 & 60 & 2,96 \\
\hline V-103 & 24 & 10,8 & 467 & 12,3 & 533 & 6,32 & 6013 & 100 & 0,00 & 88,9 & 5,9 & 22 & 13,2 & 1,9 & 60 & 2,65 \\
\hline V-108 & 25 & 12,2 & 790 & 12,7 & 819 & 6,67 & 6378 & 98 & 0,00 & 103,1 & 5,5 & 23 & 13,5 & 1,3 & 60 & 2,27 \\
\hline V-109 & 23 & 10,4 & 536 & 13,3 & 681 & 6,31 & 6013 & 100 & 0,00 & 79,8 & 4,9 & 24 & 13,3 & 1,6 & 59 & 2,86 \\
\hline V-110 & 25 & 10,2 & 472 & 10,2 & 474 & 6,33 & 6013 & 100 & 0,00 & 101,1 & 6,9 & 19 & 12,5 & 2,2 & 59 & 2,56 \\
\hline V-112 & 25 & 12,9 & 901 & 14,2 & 988 & 6,69 & 6378 & 90 & 0,00 & 97,8 & 4,9 & 26 & 12,9 & 1,4 & 61 & 2,47 \\
\hline V-113 & 25 & 15,7 & 865 & 15,3 & 843 & 6,20 & 5610 & 98 & 0,00 & 102 & 5,3 & 32 & 13,3 & 1,6 & 60 & 2,5 \\
\hline V-115 & 23 & 18,5 & 875 & 30,4 & 1434 & 6,88 & 5610 & 93 & 0,00 & 67,4 & 3,2 & 54 & 12,9 & 1,7 & 55 & 2,77 \\
\hline V-121 & 23 & 20,8 & 676 & 38,9 & 1262 & 6,17 & 6013 & 100 & 0,00 & 53,2 & 2,7 & 75 & 13,8 & 1,4 & 12 & 2,58 \\
\hline V-127 & 16 & 13,3 & 767 & 21,0 & 1213 & 6,34 & 6013 & 82 & 0,00 & 64,5 & 3,2 & 44 & 13,1 & 1,7 & 59 & 2,18 \\
\hline V-128 & 24 & 22,6 & 843 & 33,8 & 1261 & 6,25 & 6454 & 91 & 0,00 & 67,1 & 3,3 & 66 & 12,3 & 2,1 & 59 & 2,21 \\
\hline V-130 & 23 & 16,8 & 603 & 25,2 & 905 & 6,37 & 6013 & 100 & 0,00 & 68,2 & 3,8 & 49 & 13,6 & 1,3 & 56 & 2,33 \\
\hline V-131 & 19 & 13,1 & 949 & 22,2 & 1608 & 7,09 & 6454 & 82 & 0,00 & 67,3 & 3 & 40 & 12,8 & 1,7 & 59 & 2,19 \\
\hline V-132 & 13 & 7,0 & 188 & 12,7 & 339 & 6,44 & 5610 & 92 & 0,00 & 57,5 & 5,4 & 24 & 14,0 & 1,3 & 14 & 2,3 \\
\hline V-133 & 25 & 8,9 & 629 & 14,2 & 1004 & 6,00 & 6378 & 98 & 0,00 & 60,5 & 3,3 & 29 & 13,5 & 1,5 & 52 & 2,17 \\
\hline V-135 & 25 & 21,3 & 928 & 27,3 & 1191 & 6,23 & 6013 & 99 & 0,00 & 77,9 & 3,7 & 51 & 13,1 & 1,7 & 59 & 2,53 \\
\hline V-136 & 24 & 15,9 & 894 & 18,0 & 1011 & 6,29 & 6013 & 96 & 0,00 & 89,3 & 4,5 & 33 & 13,2 & 1,7 & 60 & 2,33 \\
\hline V-137 & 21 & 17,3 & 978 & 18,3 & 1038 & 6,16 & 6013 & 99 & 0,00 & 93,2 & 4,5 & 34 & 13,0 & 1,9 & 67 & 2,64 \\
\hline V-138 & 25 & 8,1 & 248 & 12,3 & 377 & 6,74 & 6094 & 100 & 0,00 & 71,3 & 6 & 25 & 13,9 & 1,6 & 18 & 2,8 \\
\hline V-139 & 25 & 14,8 & 704 & 27,0 & 1286 & 6,27 & 6013 & 88 & 0,00 & 55,2 & 2,8 & 54 & 13,7 & 1,0 & 60 & 2,43 \\
\hline V-141 & 25 & 11,6 & 1039 & 21,4 & 1914 & 6,25 & 6013 & 98 & 0,00 & 54,6 & 2,4 & 41 & 13,5 & 1,1 & 60 & 2,14 \\
\hline V-143 & 23 & 15,9 & 778 & 27,7 & 1353 & 6,26 & 6013 & 87 & 0,00 & 57,9 & 2,8 & 51 & 13,9 & 1,2 & 61 & 2,49 \\
\hline V-144 & 24 & 12,6 & 954 & 19,5 & 1482 & 6,21 & 6013 & 90 & 0,00 & 64,2 & 3 & 41 & 13,5 & 1,4 & 60 & 2,06 \\
\hline V-145 & 24 & 15,2 & 843 & 18,5 & 1026 & 6,38 & 6013 & 97 & 0,00 & 84,1 & 4,2 & 35 & 13,2 & 1,7 & 61 & 2,5 \\
\hline V-146 & 25 & 14,0 & 946 & 21,5 & 1446 & 6,55 & 6378 & 100 & 0,00 & 68,9 & 3,2 & 40 & 13,6 & 1,5 & 59 & 2,44 \\
\hline V-147 & 25 & 18,2 & 1065 & 31,6 & 1843 & 7,72 & 6454 & 98 & 0,00 & 71,7 & 3,1 & 51 & 12,9 & 1,1 & 60 & 2,12 \\
\hline V-148 & 25 & 14,7 & 885 & 25,1 & 1509 & 6,22 & 6013 & 98 & 0,00 & 58,6 & 2,7 & 50 & 13,6 & 1,3 & 46 & 2,28 \\
\hline V-151 & 25 & 10,9 & 572 & 20,7 & 1088 & 6,72 & 6454 & 97 & 0,00 & 56,9 & 3,1 & 37 & 13,0 & 1,6 & 60 & 2,06 \\
\hline V-154 & 21 & 20,5 & 1117 & 31,9 & 1739 & 6,83 & 6454 & 83 & 0,00 & 70,5 & 3 & 54 & 13,4 & 1,4 & 61 & 2,1 \\
\hline V-155 & 24 & 5,0 & 438 & 8,1 & 709 & 6,58 & 6094 & 100 & 0,00 & 65,3 & 4,2 & 15 & 14,1 & 1,5 & 60 & 3,32 \\
\hline V-156 & 23 & 15,4 & 896 & 28,1 & 1629 & 6,98 & 6454 & 99 & 0,00 & 61,8 & 2,8 & 49 & 13,2 & 1,7 & 60 & 2,1 \\
\hline V-157 & 23 & 18,9 & 840 & 29,4 & 1308 & 7,25 & 6454 & 100 & 0,00 & 74,8 & 3,6 & 47 & 13,3 & 1,3 & 60 & 2,21 \\
\hline V-158 & 21 & 12,5 & 561 & 25,8 & 1155 & 7,40 & 6454 & 97 & 0,00 & 57,8 & 3,2 & 42 & 13,2 & 1,1 & 60 & 2,04 \\
\hline V-159 & 22 & 17,2 & 689 & 31,6 & 1264 & 7,65 & 6454 & 100 & 0,00 & 67 & 3,4 & 49 & 13,5 & 0,9 & 60 & 2,03 \\
\hline V-160 & 23 & 15,1 & 839 & 26,5 & 1474 & 6,93 & 6454 & 97 & 0,00 & 63,5 & 3 & 45 & 13,3 & 1,3 & 60 & 2,13 \\
\hline V-161 & 24 & 18,7 & 1003 & 31,9 & 1712 & 6,67 & 6454 & 88 & 0,00 & 62,8 & 2,8 & 57 & 13,1 & 1,3 & 55 & 2,15 \\
\hline V-162 & 24 & 18,8 & 1085 & 35,7 & 2065 & 6,88 & 6454 & 100 & 0,00 & 58,2 & 2,5 & 60 & 13,1 & 1,5 & 60 & 2,11 \\
\hline V-163 & 22 & 17,0 & 987 & 31,5 & 1824 & 7,61 & 6454 & 94 & 0,00 & 66,2 & 2,9 & 48 & 13,5 & 1,2 & 60 & 2,07 \\
\hline V-165 & 22 & 18,0 & 1470 & 18,8 & 1543 & 6,78 & 6454 & 94 & 0,00 & 103,5 & 4,3 & 35 & 12,2 & 1,7 & 60 & 2,03 \\
\hline V-166 & 22 & 16,2 & 1073 & 17,2 & 1135 & 7,30 & 6454 & 99 & 0,00 & 110,5 & 5,2 & 29 & 12,9 & 1,5 & 60 & 2,08 \\
\hline V-167 & 22 & 23,2 & 1308 & 25,9 & 1464 & 7,46 & 6454 & 80 & 0,01 & 106,8 & 4,6 & 43 & 13,0 & 1,7 & 64 & 2,09 \\
\hline V-168 & 24 & 18,1 & 949 & 25,3 & 1325 & 6,75 & 5610 & 99 & 0,00 & 77,7 & 3,7 & 43 & 13,1 & 1,4 & 60 & 2,79 \\
\hline V-169 & 23 & 20,5 & 1018 & 22,2 & 1102 & 7,67 & 6454 & 98 & 0,00 & 113,4 & 5,4 & 36 & 12,6 & 1,7 & 60 & 2,03 \\
\hline V-171 & 5 & 5,9 & 75 & 5,0 & 64 & 6,57 & 5610 & 99 & 0,00 & 123,2 & 21,1 & 10 & & & & 3,39 \\
\hline V-172 & 23 & 23,6 & 1488 & 28,6 & 1806 & 7,14 & 6454 & 92 & 0,00 & 94,4 & 3,8 & 47 & 13,3 & 1,3 & 60 & 2,13 \\
\hline V-173 & 24 & 20,5 & 940 & 21,7 & 994 & 7,19 & 6454 & 98 & 0,00 & 109 & 5,4 & 36 & 12,9 & 1,4 & 60 & 2,08 \\
\hline V-183 & 24 & 12,5 & 535 & 20,5 & 879 & 7,51 & 6454 & 90 & 0,01 & 73,4 & 4,3 & 34 & 13,3 & 1,3 & 60 & 2,1 \\
\hline V-191 & 25 & 20,8 & 1128 & 28,8 & 1559 & 7,04 & 6454 & 100 & 0,00 & 81,8 & 3,6 & 47 & 13,0 & 1,5 & 60 & 2,12 \\
\hline V-192 & 25 & 21,8 & 864 & 22,7 & 901 & 6,02 & 5610 & 100 & 0,00 & 92,7 & 4,8 & 45 & 13,4 & 1,4 & 34 & 2,84 \\
\hline V-194 & 24 & 14,5 & 316 & 11,8 & 257 & 6,34 & 5610 & 100 & 0,00 & 124,7 & 10,8 & 22 & 13,1 & 1,2 & 24 & 2,68 \\
\hline V-197 & 18 & 3,8 & 232 & 2,0 & 120 & 6,78 & 6387 & 100 & 0,00 & 208,4 & 23,8 & 3 & 13,8 & 1,1 & 13 & 3,47 \\
\hline V-201 & 22 & 19,8 & 1226 & 18,4 & 1139 & 6,16 & 5610 & 100 & 0,00 & 106,2 & 4,9 & 36 & 13,1 & 1,7 & 60 & 2,18 \\
\hline V-203 & 22 & 23,3 & 846 & 36,1 & 1312 & 6,63 & 5610 & 100 & 0,00 & 68,7 & 3,3 & 67 & 13,2 & 1,5 & 60 & 2,51 \\
\hline V-204 & 23 & 20,6 & 859 & 26,4 & 1101 & 6,07 & 5610 & 98 & 0,00 & 76 & 3,8 & 52 & 13,6 & 1,2 & 60 & 2,4 \\
\hline V-206 & 17 & 17,3 & 562 & 21,7 & 703 & 6,63 & 6094 & 97 & 0,00 & 85,1 & 5,1 & 43 & 13,9 & 1,3 & 20 & 2,79 \\
\hline V-207 & 21 & 18,5 & 482 & 22,1 & 576 & 6,68 & 6387 & 100 & 0,00 & 89,7 & 5,8 & 39 & 12,8 & 2,0 & 25 & 2,43 \\
\hline$V-216$ & 15 & 9,5 & 186 & 18,5 & 363 & 6,82 & 6094 & 95 & 0,00 & 56,2 & 5,2 & 31 & 13,1 & 1,5 & 7 & 2,4 \\
\hline V-217 & 23 & 16,1 & 843 & 18,0 & 939 & 6,69 & 6094 & 76 & 0,01 & 96,3 & 4,9 & 32 & 12,6 & 1,9 & 60 & 2,6 \\
\hline
\end{tabular}




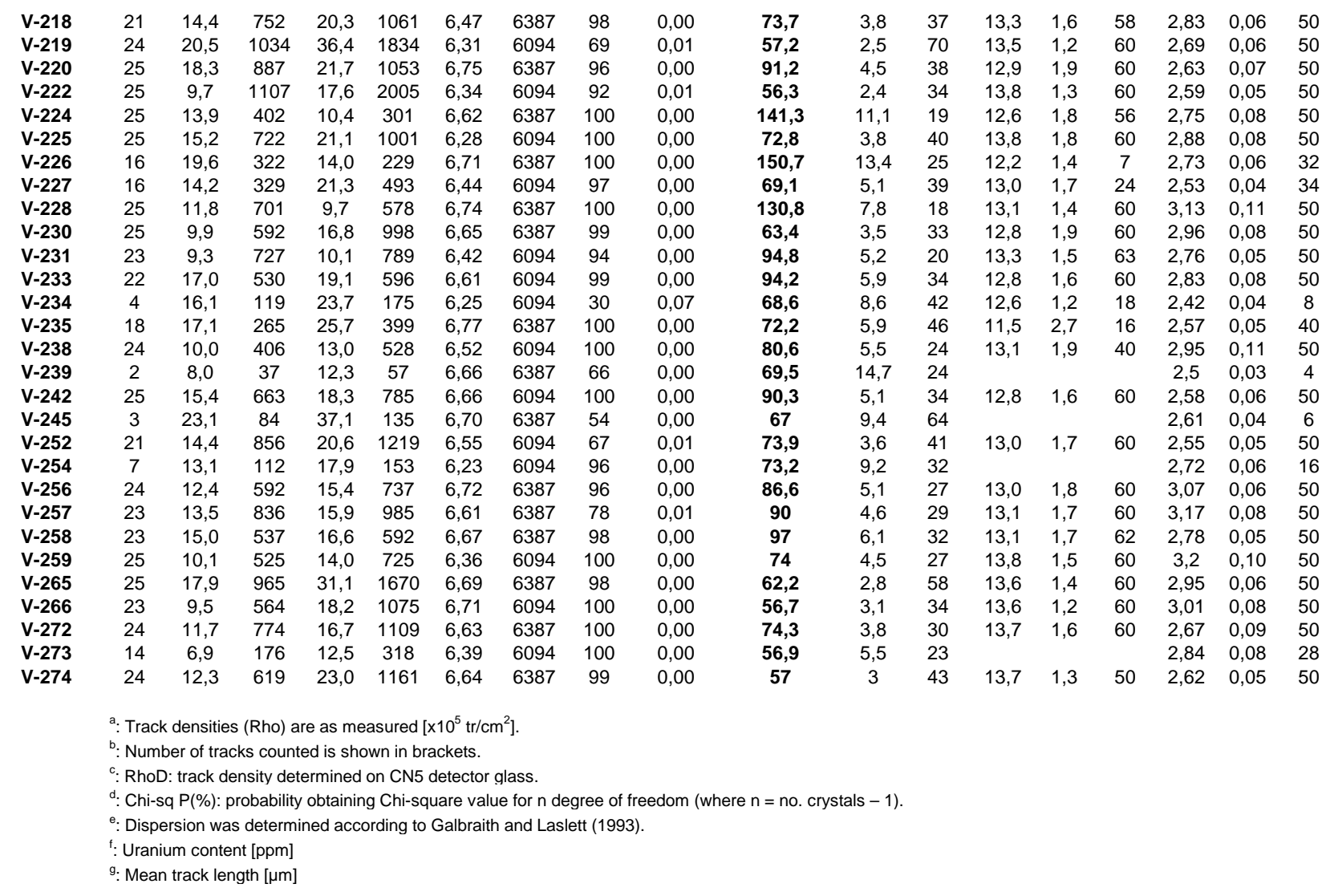



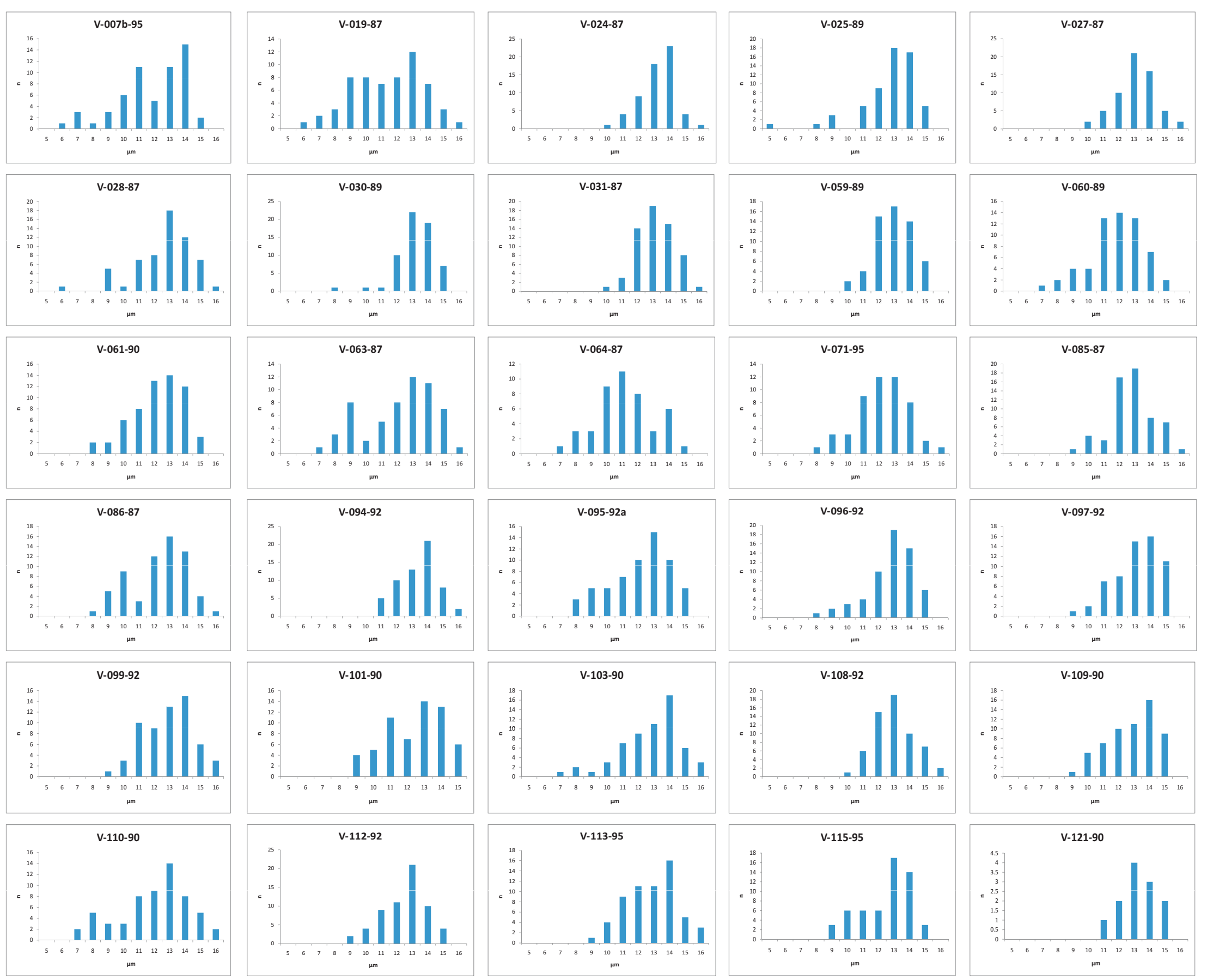

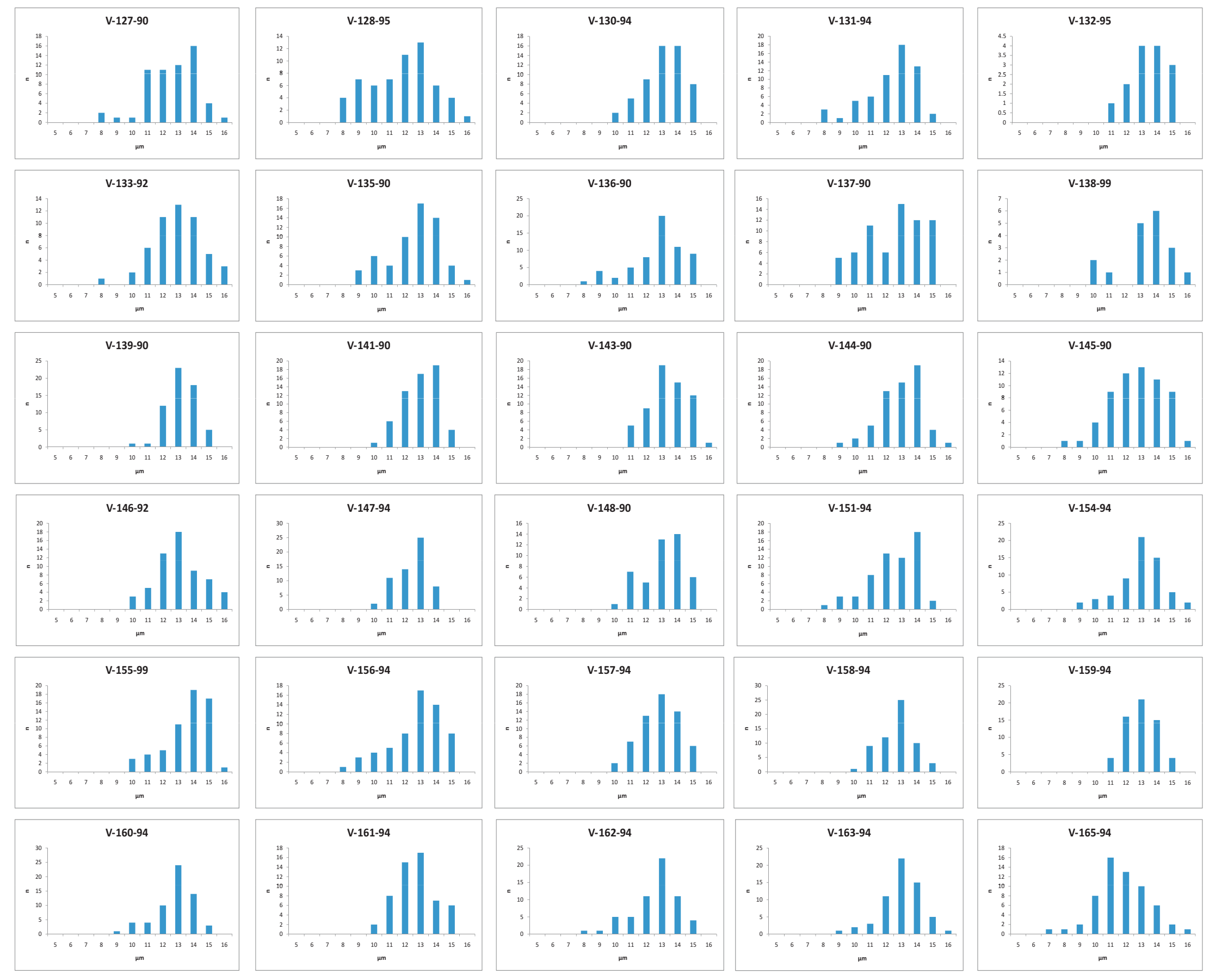


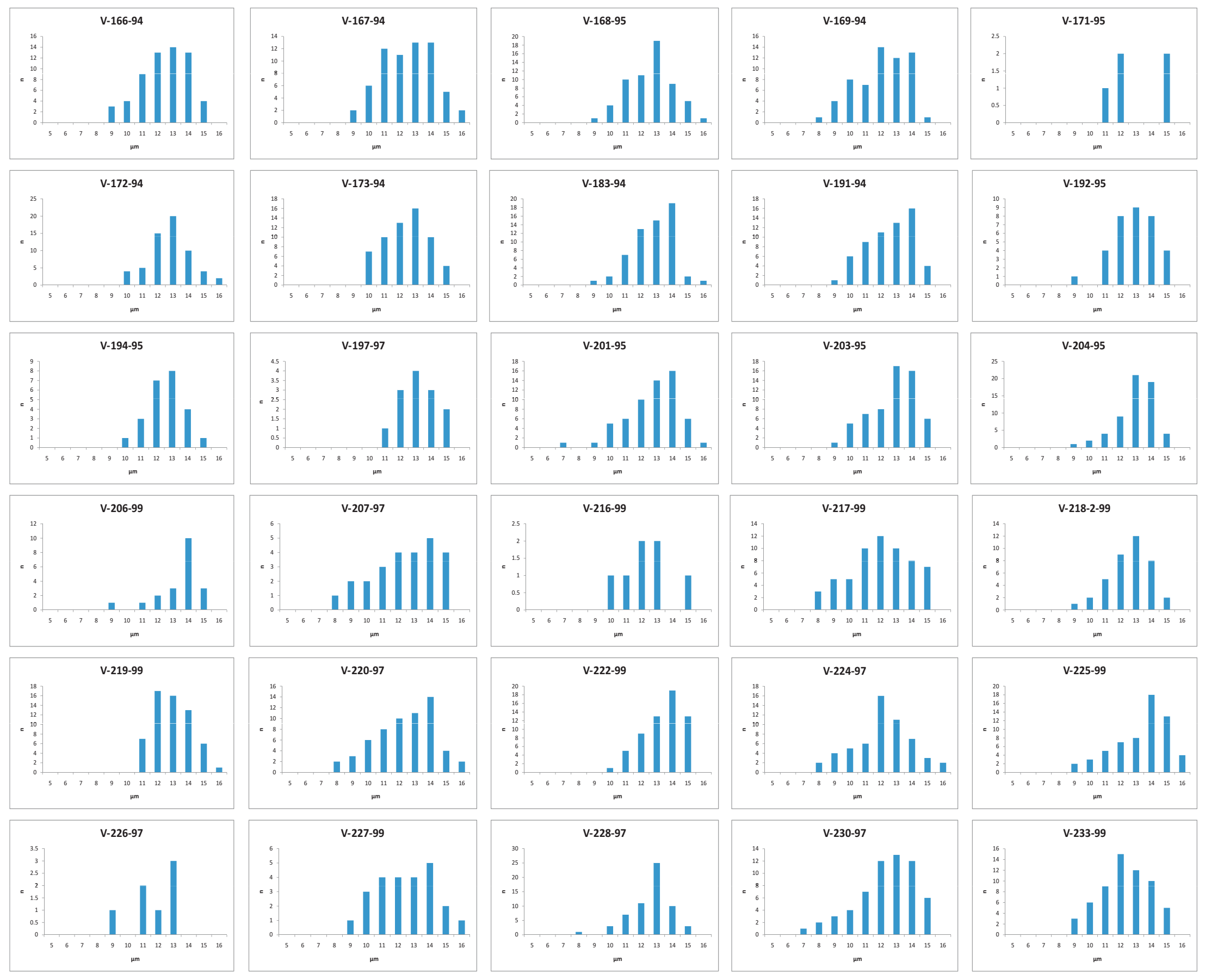




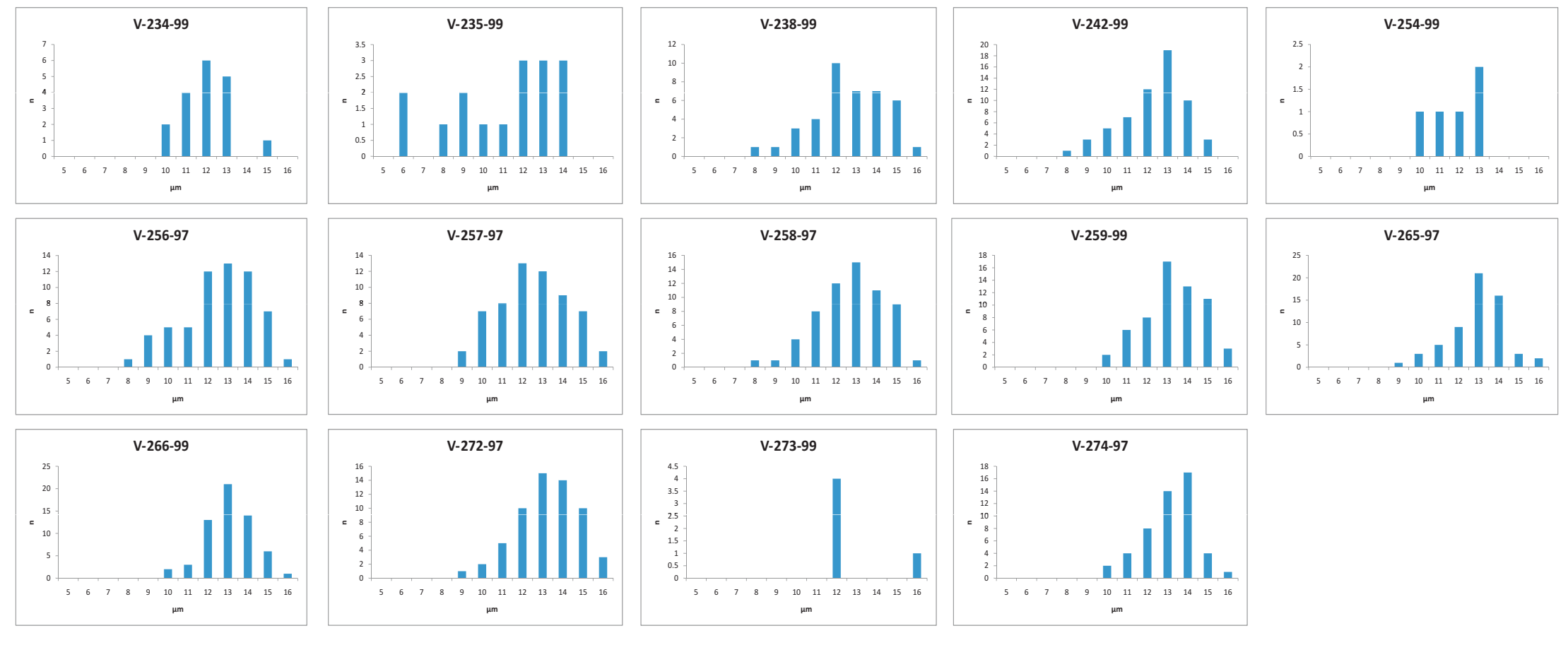




\begin{tabular}{|c|c|c|c|c|c|c|c|c|c|c|c|c|c|c|c|}
\hline & & & & & U238 & & & Th232 & & & & $\mathrm{Sm}$ & & Ejection & \\
\hline & & vol. $^{a}$ & $\pm 1 \mathrm{~s}$ & mass $^{b}$ & $\pm 1 s$ & conc. & mass $^{b}$ & $\pm 1 \mathrm{~s}$ & conc. & $\mathrm{Th} / \mathrm{U}$ & mass $^{b}$ & $\pm 1 \mathrm{~s}$ & conc. & correct. $^{\mathrm{c}}$ & He-age \\
\hline Sample & aliq. & [ncc] & [\%] & [ng] & [\%] & [ppm] & [ng] & [\%] & [ppm] & ratio & [ng] & [\%] & [ppm] & $(\mathrm{Ft})$ & [Ma] \\
\hline V-4 & $\# 1$ & 1,711 & 1,7 & 0,154 & 1,8 & 14 & 0,555 & 2,4 & 51 & 3,61 & 0,67 & 10,1 & 61 & 0,751 & 48,6 \\
\hline & \#2 & 2,106 & 1,7 & 0,183 & 1,8 & 101 & 0,703 & 2,4 & 386 & 3,84 & 1,20 & 9,8 & 658 & 0,735 & 48,4 \\
\hline & $\# 3$ & 0,395 & 5,0 & 0,072 & 1,9 & 46 & 0,120 & 2,5 & 76 & 1,66 & 1,03 & 9,6 & 652 & 0,744 & 29,9 \\
\hline V-19 & $\# 1$ & 1,461 & 1,7 & 0,153 & 4,0 & 81 & 0,173 & 4,0 & 92 & 1,13 & & & & 0,708 & 62,2 \\
\hline & \#2 & 2,031 & 1,7 & 0,251 & 4,0 & 158 & 0,009 & 4,0 & 6 & 0,04 & & & & 0,687 & 66,1 \\
\hline & $\# 3$ & 1,070 & 1,7 & 0,149 & 4,0 & 68 & 0,202 & 4,0 & 92 & 1,35 & & & & 0,711 & 44,8 \\
\hline V-25 & $\# 1$ & 0,737 & 1,2 & 0,118 & 1,9 & 41 & 0,033 & 2,5 & 11 & 0,28 & 0,60 & 2,4 & 210 & 0,749 & 46,5 \\
\hline & \#2 & 0,721 & 1,1 & 0,122 & 1,9 & 50 & 0,029 & 2,5 & 12 & 0,24 & 0,51 & 2,4 & 212 & 0,715 & 44,9 \\
\hline & \#3 & 1,318 & 1,1 & 0,206 & 1,8 & 84 & 0,030 & 2,6 & 12 & 0,15 & 0,48 & 2,4 & 196 & 0,740 & 50,1 \\
\hline V-27 & $\# 1$ & 1,833 & 1,1 & 0,268 & 1,8 & 53 & 0,014 & 4,3 & 3 & 0,05 & 1,21 & 2,4 & 238 & 0,794 & 53,8 \\
\hline & \#2 & 1,308 & 1,1 & 0,203 & 1,8 & 73 & 0,004 & 2,8 & 1 & 0,02 & 0,61 & 2,4 & 222 & 0,723 & 51,7 \\
\hline & \#3 & 5,042 & 1,0 & 0,728 & 1,8 & 192 & 0,006 & 2,8 & 2 & 0,01 & 0,77 & 2,4 & 202 & 0,795 & 56,5 \\
\hline & $\# 4$ & 1,154 & 1,1 & 0,178 & 1,8 & 73 & 0,016 & 3,1 & 7 & 0,09 & 0,25 & 2,4 & 104 & 0,756 & 51,8 \\
\hline V-28 & $\# 1$ & 0,585 & 1,8 & 0,079 & 4,0 & 57 & 0,056 & 4,0 & 41 & 0,72 & & & & 0,684 & 52,4 \\
\hline & \#2 & 0,804 & 1,8 & 0,109 & 4,0 & 106 & 0,078 & 4,0 & 75 & 0,71 & & & & 0,615 & 51,8 \\
\hline & $\# 3$ & 0,552 & 1,8 & 0,093 & 4,0 & 83 & 0,046 & 4,0 & 41 & 0,50 & & & & 0,649 & 43,9 \\
\hline V-29 & $\# 3$ & 0,349 & 1,8 & 0,063 & 2,1 & 69 & 0,002 & 14,3 & 3 & 0,04 & 0,19 & 3,4 & 207 & 0,623 & 43,9 \\
\hline & \#4 & 0,433 & 1,2 & 0,042 & 2,4 & 16 & 0,075 & 2,4 & 29 & 1,79 & 2,32 & 6,0 & 898 & 0,629 & 45,6 \\
\hline & $\# 5$ & 0,574 & 1,1 & 0,074 & 2,0 & 44 & 0,103 & 2,4 & 61 & 1,38 & 1,32 & 6,0 & 785 & 0,617 & 43,3 \\
\hline V-30 & $\# 1$ & 2,670 & 1,7 & 0,437 & 4,0 & 279 & 0,136 & 4,0 & 87 & 0,31 & & & & 0,717 & 47,0 \\
\hline & \#3 & 0,344 & 1,9 & 0,041 & 4,0 & 44 & 0,108 & 4,0 & 117 & 2,66 & & & & 0,644 & 42,9 \\
\hline V-31 & $\# 1$ & 1,353 & 1,1 & 0,214 & 1,8 & 50 & 0,012 & 2,9 & 3 & 0,06 & 0,93 & 2,4 & 219 & 0,743 & 49,7 \\
\hline & \#2 & 0,843 & 1,2 & 0,119 & 1,9 & 30 & 0,007 & 2,6 & 2 & 0,06 & 0,74 & 2,4 & 187 & 0,771 & 55,0 \\
\hline & $\# 3$ & 2,499 & 1,1 & 0,404 & 1,8 & 111 & 0,010 & 2,8 & 3 & 0,02 & 0,76 & 2,4 & 209 & 0,782 & 49,9 \\
\hline V-59 & \#2 & 0,499 & 0,9 & 0,097 & 3,0 & 55 & 0,031 & 3,3 & 18 & 0,32 & 0,42 & 3,4 & 237 & 0,787 & 38,2 \\
\hline & \#3 & 0,160 & 2,2 & 0,034 & 2,3 & 14 & 0,011 & 3,2 & 5 & 0,33 & 0,39 & 6,0 & 159 & 0,560 & 33,2 \\
\hline & $\# 4$ & 1,709 & 1,7 & 0,315 & 1,8 & 112 & 0,058 & 2,5 & 21 & 0,18 & 0,91 & 5,9 & 323 & 0,670 & 42,0 \\
\hline & $\# 5$ & 0,776 & 1,3 & 0,138 & 1,9 & 120 & 0,025 & 2,6 & 22 & 0,18 & 0,32 & 2,4 & 280 & 0,699 & 43,7 \\
\hline V-60 & $\# 1$ & 0,109 & 2,0 & 0,021 & 6,0 & 11 & 0,034 & 3,1 & 18 & 1,62 & 0,32 & 5,8 & 168 & 0,685 & 28,7 \\
\hline & \#2 & 0,223 & 1,4 & 0,042 & 3,7 & 37 & 0,013 & 4,2 & 12 & 0,32 & 0,08 & 6,2 & 73 & 0,686 & 39,8 \\
\hline & $\# 4$ & 0,172 & 1,5 & 0,034 & 4,3 & 14 & 0,024 & 3,4 & 10 & 0,70 & 0,12 & 5,6 & 48 & 0,722 & 34,9 \\
\hline & $\# 5$ & 0,047 & 3,0 & 0,013 & 11,6 & 14 & 0,011 & 4,5 & 13 & 0,89 & 0,35 & 5,9 & 395 & 0,678 & 21,4 \\
\hline & $\# 6$ & 0,074 & 2,6 & 0,014 & 9,4 & 17 & 0,023 & 3,5 & 27 & 1,61 & 0,50 & 5,9 & 605 & 0,614 & 25,9 \\
\hline V-63 & $\# 1$ & 1,092 & 1,1 & 0,194 & 1,8 & 85 & 0,043 & 2,5 & 19 & 0,22 & 0,45 & 2,4 & 195 & 0,703 & 43,5 \\
\hline & \#2 & 1,153 & 1,6 & 0,200 & 1,8 & 107 & 0,025 & 2,5 & 13 & 0,12 & 0,44 & 3,6 & 233 & 0,730 & 45,4 \\
\hline & $\# 3$ & 1,811 & 1,1 & 0,302 & 1,8 & 102 & 0,045 & 2,5 & 15 & 0,15 & 0,69 & 2,4 & 234 & 0,760 & 46,9 \\
\hline & $\# 4$ & 2,955 & 1,6 & 0,461 & 1,8 & 218 & 0,053 & 2,4 & 25 & 0,11 & 0,88 & 3,6 & 416 & 0,678 & 50,8 \\
\hline & $\# 5$ & 0,497 & 1,3 & 0,098 & 1,9 & 67 & 0,021 & 2,6 & 14 & 0,21 & 0,29 & 2,4 & 198 & 0,675 & 39,0 \\
\hline V-64 & $\# 2$ & 0,409 & 1,8 & 0,066 & 2,0 & 37 & 0,029 & 2,5 & 16 & 0,44 & 0,33 & 3,6 & 183 & 0,732 & 44,4 \\
\hline & $\# 3$ & 1,717 & 1,6 & 0,269 & 1,8 & 63 & 0,064 & 2,5 & 15 & 0,24 & 1,18 & 3,6 & 277 & 0,801 & 48,2 \\
\hline & $\# 4$ & 0,287 & 2,0 & 0,040 & 2,2 & 17 & 0,111 & 2,4 & 47 & 2,74 & 0,28 & 3,6 & 119 & 0,723 & 34,5 \\
\hline V-108 & $\# 1$ & 0,569 & 1,8 & 0,041 & 2,2 & 11 & 0,159 & 2,4 & 43 & 3,88 & 0,42 & 5,6 & 115 & 0,731 & 57,3 \\
\hline & \#2 & 0,921 & 1,7 & 0,068 & 1,9 & 12 & 0,279 & 2,4 & 48 & 4,11 & 0,71 & 5,5 & 121 & 0,776 & 54,4 \\
\hline & $\# 3$ & 0,303 & 1,9 & 0,036 & 2,2 & 13 & 0,060 & 2,5 & 21 & 1,64 & 0,59 & 5,4 & 205 & 0,700 & 45,0 \\
\hline V-121 & $\# 1$ & 0,533 & 1,3 & 0,093 & 1,9 & 79 & 0,022 & 2,7 & 19 & 0,24 & 0,55 & 2,4 & 464 & 0,796 & 42,7 \\
\hline & \#2 & 0,436 & 1,3 & 0,072 & 2,0 & 48 & 0,004 & 4,4 & 2 & 0,05 & 0,35 & 6,0 & 232 & 0,598 & 47,2 \\
\hline & $\# 3$ & 1,253 & 1,0 & 0,184 & 1,8 & 138 & 0,035 & 2,5 & 26 & 0,19 & 0,49 & 6,0 & 366 & 0,743 & 52,6 \\
\hline V-135 & $\# 1$ & 1,323 & 1,5 & 0,166 & 1,9 & 41 & 0,034 & 2,7 & 8 & 0,20 & 1,56 & 8,2 & 383 & 0,765 & 58,4 \\
\hline & $\# 2$ & 0,226 & 2,0 & 0,023 & 3,7 & 11 & 0,060 & 2,5 & 29 & 2,64 & 0,58 & 8,2 & 279 & 0,575 & 44,6 \\
\hline & $\# 3$ & 0,623 & 1,6 & 0,090 & 2,0 & 62 & 0,038 & 2,6 & 26 & 0,42 & 0,53 & 9,0 & 358 & 0,567 & 49,7 \\
\hline V-136 & $\# 1$ & 0,453 & 1,8 & 0,071 & 5,5 & 49 & 0,027 & 13,0 & 19 & 0,39 & 0,50 & 3,6 & 345 & 0,679 & 46,0 \\
\hline & $\# 2$ & 0,444 & 1,8 & 0,052 & 2,1 & 31 & 0,020 & 3,2 & 12 & 0,38 & 0,85 & 3,6 & 502 & 0,754 & 57,5 \\
\hline V-138 & $\# 1$ & 0,063 & 2,8 & 0,005 & 9,4 & 3 & 0,025 & 2,7 & 17 & 5,06 & 0,35 & 4,2 & 229 & 0,664 & 37,5 \\
\hline & \#2 & 0,284 & 1,9 & 0,021 & 2,9 & 5 & 0,127 & 2,5 & 33 & 6,13 & 1,08 & 3,7 & 278 & 0,716 & 39,3 \\
\hline & $\# 3$ & 0,137 & 2,3 & 0,005 & 10,0 & 2 & 0,051 & 2,5 & 20 & 10,59 & 0,80 & 3,6 & 312 & 0,740 & 48,3 \\
\hline V-139 & $\# 1$ & 1,036 & 1,7 & 0,177 & 1,8 & 112 & 0,021 & 2,8 & 13 & 0,12 & 0,50 & 3,5 & 316 & 0,637 & 46,1 \\
\hline & \#2 & 0,747 & 1,8 & 0,131 & 1,9 & 81 & 0,031 & 2,6 & 19 & 0,24 & 0,59 & 3,4 & 365 & 0,539 & 43,2 \\
\hline & \#3 & 0,233 & 2,1 & 0,028 & 2,5 & 10 & 0,075 & 2,5 & 27 & 2,66 & 1,14 & 3,7 & 410 & 0,692 & 34,8 \\
\hline & $\# 4$ & 0,376 & 1,9 & 0,058 & 2,0 & 19 & 0,026 & 2,7 & 8 & 0,45 & 0,68 & 6,9 & 219 & 0,666 & 44,8 \\
\hline V-144 & $\# 1$ & 0,475 & 1,4 & 0,056 & 2,0 & 22 & 0,202 & 2,4 & 78 & 3,58 & 0,71 & 2,4 & 277 & 0,643 & 35,7 \\
\hline & \#2 & 0,519 & 1,3 & 0,051 & 2,1 & 22 & 0,121 & 2,4 & 51 & 2,38 & 0,34 & 2,4 & 146 & 0,688 & 52,0 \\
\hline & \#3 & 0,090 & 2,4 & 0,017 & 4,2 & 6 & 0,018 & 2,6 & 6 & 1,04 & 0,05 & 2,4 & 17 & 0,849 & 34,4 \\
\hline & $\# 4$ & 0,517 & 1,4 & 0,096 & 1,9 & 61 & 0,043 & 2,5 & 27 & 0,45 & 0,57 & 2,4 & 361 & 0,674 & 38,5 \\
\hline V-145 & $\# 1$ & 0,608 & 1,3 & 0,104 & 1,9 & 61 & 0,021 & 3,6 & 12 & 0,20 & 0,51 & 2,4 & 299 & 0,614 & 44,4 \\
\hline & \#2 & 0,439 & 1,4 & 0,092 & 1,9 & 65 & 0,031 & 2,5 & 22 & 0,34 & 0,47 & 2,4 & 335 & 0,591 & 35,0 \\
\hline & \#3 & 0,330 & 1,4 & 0,038 & 2,3 & 62 & 0,014 & 2,7 & 23 & 0,38 & 0,19 & 2,4 & 309 & 0,720 & 63,0 \\
\hline & $\# 4$ & 0,807 & 1,2 & 0,126 & 1,9 & 56 & 0,033 & 2,5 & 15 & 0,26 & 0,60 & 2,4 & 269 & 0,743 & 47,9 \\
\hline V-155 & $\# 1$ & 0,194 & 2,2 & 0,017 & 4,2 & 10 & 0,077 & 2,6 & 45 & 4,61 & 0,76 & 5,0 & 447 & 0,671 & 38,8 \\
\hline & \#2 & 0,231 & 2,1 & 0,015 & 4,5 & 6 & 0,061 & 2,7 & 25 & 3,95 & 0,92 & 4,8 & 373 & 0,725 & 51,2 \\
\hline & $\# 3$ & 0,112 & 2,6 & 0,009 & 7,7 & 10 & 0,038 & 2,9 & 44 & 4,51 & 0,33 & 5,0 & 374 & 0,623 & 45,4 \\
\hline V-156 & $\# 1$ & 1,345 & 1,1 & 0,187 & 1,9 & 54 & 0,008 & 17,4 & 2 & 0,04 & 1,03 & 3,4 & 297 & 0,731 & 56,3 \\
\hline & \#2 & 1,115 & 1,1 & 0,155 & 1,8 & 63 & 0,007 & 3,0 & 3 & 0,04 & 0,86 & 3,4 & 347 & 0,753 & 56,4 \\
\hline & $\# 3$ & 0,784 & 1,2 & 0,117 & 1,9 & 53 & 0,007 & 3,0 & 3 & 0,06 & 0,65 & 3,4 & 295 & 0,759 & 52,4 \\
\hline V-157 & $\# 1$ & 0,296 & 1,9 & 0,040 & 2,3 & 30 & 0,117 & 2,4 & 86 & 2,93 & 0,08 & 3,6 & 61 & 0,699 & 36,0 \\
\hline & \#3 & 0,761 & 1,7 & 0,125 & 1,9 & 60 & 0,076 & 2,4 & 37 & 0,61 & 0,48 & 3,6 & 233 & 0,714 & 42,9 \\
\hline & $\# 4$ & 0,435 & 1,8 & 0,083 & 1,9 & 67 & 0,013 & 3,2 & 11 & 0,16 & 0,27 & 3,6 & 220 & 0,664 & 40,6 \\
\hline & $\# 5$ & 0,840 & 1,7 & 0,140 & 1,8 & 120 & 0,101 & 2,4 & 87 & 0,72 & 0,46 & 3,6 & 398 & 0,658 & 41,3 \\
\hline V-161 & $\# 1$ & 0,263 & 1,9 & 0,045 & 2,1 & 36 & 0,004 & 2,5 & 3 & 0,10 & 0,30 & 3,6 & 240 & 0,644 & 44,7 \\
\hline & \#3 & 0,727 & 1,1 & 0,113 & 1,9 & 89 & 0,006 & 2,6 & 5 & 0,06 & 0,43 & 6,0 & 339 & 0,624 & 50,7 \\
\hline & $\# 4$ & 0,394 & 1,2 & 0,052 & 2,1 & 41 & 0,002 & 21,0 & 2 & 0,04 & 0,26 & 6,0 & 203 & 0,589 & 59,0 \\
\hline
\end{tabular}




\begin{tabular}{|c|c|c|c|c|c|c|c|c|c|c|c|c|c|c|c|c|c|c|c|}
\hline & $\# 5$ & 0,656 & 1,1 & 0,115 & 1,9 & 76 & 0,003 & 6,9 & 2 & 0,03 & 0,40 & 6,0 & 267 & 0,608 & 45,6 & 75,1 & 4,7 & 81,5 & 6,7 \\
\hline \multirow[t]{3}{*}{ V-163 } & $\# 1$ & 0,905 & 1,1 & 0,140 & 1,9 & 57 & 0,013 & 2,8 & 5 & 0,09 & 0,51 & 2,4 & 208 & 0,703 & 50,9 & 72,3 & 3,5 & & \\
\hline & \#2 & 0,091 & 2,0 & 0,016 & 4,2 & 15 & 0,010 & 4,8 & 9 & 0,61 & 0,04 & 2,4 & 32 & 0,636 & 40,0 & 62,9 & 4,3 & & \\
\hline & \#3 & 0,231 & 1,5 & 0,056 & 2,0 & 78 & 0,005 & 2,7 & 8 & 0,10 & 0,15 & 2,4 & 206 & 0,600 & 32,8 & 54,8 & 3,5 & 63,3 & 5,1 \\
\hline \multirow[t]{3}{*}{ V-165 } & $\# 1$ & 0,887 & 1,7 & 0,091 & 1,9 & 44 & 0,108 & 2,4 & 52 & 1,19 & 1,28 & 3,6 & 614 & 0,659 & 57,6 & 87,4 & 4,9 & & \\
\hline & \#2 & 2,103 & 1,6 & 0,247 & 1,8 & 72 & 0,159 & 2,4 & 46 & 0,64 & 1,55 & 3,6 & 449 & 0,725 & 58,3 & 80,4 & 3,8 & & \\
\hline & $\# 3$ & 1,314 & 1,7 & 0,122 & 1,9 & 29 & 0,386 & 2,4 & 92 & 3,15 & 2,69 & 3,6 & 644 & 0,812 & 46,0 & 56,7 & 2,0 & 74,8 & 9,3 \\
\hline \multirow[t]{3}{*}{ V-166 } & \#2 & 1,247 & 1,1 & 0,137 & 1,9 & 36 & 0,058 & 2,4 & 15 & 0,43 & 1,01 & 3,4 & 268 & 0,783 & 64,6 & 82,5 & 3,1 & & \\
\hline & \#3 & 0,596 & 1,3 & 0,073 & 2,0 & 19 & 0,168 & 2,4 & 43 & 2,31 & 1,84 & 3,4 & 474 & 0,762 & 38,6 & 50,6 & 2,0 & & \\
\hline & $\# 4$ & 1,192 & 1,1 & 0,103 & 1,9 & 20 & 0,178 & 2,4 & 34 & 1,73 & 1,96 & 3,4 & 380 & 0,765 & 60,8 & 79,5 & 3,1 & 70,9 & 10,2 \\
\hline \multirow[t]{4}{*}{ V-167 } & $\# 1$ & 0,478 & 1,8 & 0,032 & 2,6 & 12 & 0,131 & 2,4 & 51 & 4,13 & 1,80 & 3,6 & 707 & 0,697 & 50,8 & 73,0 & 3,7 & & \\
\hline & \#2 & 0,814 & 1,7 & 0,090 & 1,9 & 47 & 0,012 & 2,7 & 6 & 0,13 & 0,53 & 3,6 & 279 & 0,717 & 69,2 & 96,5 & 4,7 & & \\
\hline & $\# 3$ & 1,723 & 1,6 & 0,191 & 1,8 & 32 & 0,006 & 2,6 & 1 & 0,03 & 1,13 & 3,6 & 187 & 0,803 & 70,4 & 87,6 & 3,3 & & \\
\hline & $\# 4$ & 1,347 & 1,7 & 0,131 & 1,8 & 29 & 0,148 & 2,4 & 33 & 1,12 & 0,52 & 3,6 & 114 & 0,714 & 65,0 & 91,1 & 4,4 & 87,0 & 5,0 \\
\hline \multirow[t]{4}{*}{ V-168 } & $\# 1$ & 0,298 & 1,9 & 0,044 & 2,2 & 13 & 0,006 & 2,8 & 2 & 0,14 & 0,06 & 3,6 & 17 & 0,782 & 53,3 & 68,2 & 2,9 & & \\
\hline & \#2 & 0,295 & 1,9 & 0,048 & 2,2 & 32 & 0,049 & 2,5 & 33 & 1,04 & 0,67 & 3,6 & 452 & 0,718 & 37,6 & 52,3 & 2,6 & & \\
\hline & \#3 & 0,037 & 3,5 & 0,006 & 11,7 & 1 & 0,008 & 2,8 & 2 & 1,39 & 0,35 & 3,6 & 72 & 0,779 & 29,0 & 37,2 & 2,9 & & \\
\hline & $\# 4$ & 1,281 & 1,7 & 0,163 & 1,8 & 77 & 0,003 & 10,6 & 1 & 0,02 & 0,46 & 3,6 & 215 & 0,688 & 63,2 & 91,8 & 4,8 & 62,4 & 11,7 \\
\hline \multirow[t]{3}{*}{ V-172 } & $\# 1$ & 0,510 & 1,4 & 0,044 & 2,1 & 10 & 0,084 & 2,4 & 19 & 1,91 & 0,51 & 3,4 & 112 & 0,793 & 61,6 & 77,7 & 2,9 & & \\
\hline & $\# 2$ & 3,452 & 1,0 & 0,330 & 1,8 & 71 & 0,017 & 2,8 & 4 & 0,05 & 1,47 & 3,4 & 318 & 0,810 & 82,1 & 101,4 & 3,5 & & \\
\hline & $\# 3$ & 7,097 & 1,0 & 0,780 & 1,8 & 158 & 0,011 & 2,8 & 2 & 0,01 & 1,39 & 3,4 & 282 & 0,810 & 73,6 & 90,9 & 3,2 & 90,0 & 6,8 \\
\hline \multirow[t]{3}{*}{ V-191 } & $\# 1$ & 1,413 & 1,6 & 0,201 & 1,8 & 124 & 0,007 & 3,8 & 4 & 0,03 & 0,40 & 3,6 & 248 & 0,718 & 56,7 & 79,0 & 3,8 & & \\
\hline & $\# 3$ & 2,381 & 1,6 & 0,317 & 1,8 & 159 & 0,027 & 2,5 & 13 & 0,08 & 0,57 & 3,6 & 286 & 0,752 & 59,9 & 79,6 & 3,5 & & \\
\hline & $\# 4$ & 2,047 & 1,6 & 0,275 & 1,8 & 96 & 0,043 & 2,5 & 15 & 0,16 & 1,02 & 3,6 & 357 & 0,746 & 57,6 & 77,2 & 3,4 & 78,6 & 0,7 \\
\hline \multirow[t]{3}{*}{ V-201 } & \#2 & 0,315 & 1,9 & 0,038 & 2,3 & 27 & 0,094 & 2,4 & 68 & 2,50 & 0,46 & 3,4 & 332 & 0,653 & 41,0 & 62,8 & 3,6 & & \\
\hline & $\# 3$ & 0,518 & 1,8 & 0,083 & 2,0 & 49 & 0,065 & 2,5 & 38 & 0,78 & 0,76 & 3,4 & 451 & 0,663 & 40,8 & 61,5 & 3,4 & & \\
\hline & $\# 4$ & 0,810 & 1,7 & 0,112 & 1,9 & 59 & 0,072 & 2,4 & 38 & 0,64 & 0,87 & 3,4 & 457 & 0,698 & 49,0 & 70,2 & 3,6 & 64,8 & 2,7 \\
\hline \multirow[t]{3}{*}{ V-204 } & $\# 1$ & 0,250 & 2,1 & 0,030 & 2,5 & 20 & 0,042 & 2,5 & 27 & 1,39 & 1,18 & 3,4 & 772 & 0,635 & 41,5 & 65,3 & 4,0 & & \\
\hline & \#2 & 0,380 & 1,8 & 0,038 & 2,5 & 20 & 0,023 & 2,8 & 12 & 0,61 & 0,57 & 3,4 & 296 & 0,698 & 64,6 & 92,6 & 4,9 & & \\
\hline & $\# 3$ & 0,212 & 2,0 & 0,036 & 2,6 & 40 & 0,046 & 2,5 & 52 & 1,28 & 0,59 & 3,4 & 657 & 0,594 & 33,7 & 56,8 & 3,8 & 71,6 & 10,8 \\
\hline \multirow[t]{4}{*}{ V-217 } & \#3 & 1,364 & 1,6 & 0,155 & 1,8 & 51 & 0,020 & 2,6 & 7 & 0,13 & 1,24 & 3,4 & 406 & 0,748 & 66,3 & 88,5 & 3,9 & & \\
\hline & $\# 4$ & 0,405 & 1,2 & 0,071 & 2,0 & 28 & 0,017 & 2,7 & 7 & 0,24 & 0,52 & 8,4 & 204 & 0,871 & 41,9 & 48,1 & 1,4 & & \\
\hline & $\# 5$ & 1,809 & 0,9 & 0,291 & 1,8 & 96 & 0,004 & 2,8 & 1 & 0,01 & 1,02 & 8,4 & 337 & 0,000 & 49,7 & 63,7 & 2,4 & & \\
\hline & $\# 7$ & 1,896 & 1,0 & 0,277 & 1,8 & 148 & 0,018 & 2,9 & 9 & 0,06 & 0,66 & 8,4 & 353 & 0,822 & 54,6 & 66,4 & 2,2 & 66,7 & 8,3 \\
\hline \multirow[t]{3}{*}{ V-225 } & $\# 1$ & 1,473 & 1,7 & 0,141 & 1,8 & 49 & 0,137 & 2,4 & 48 & 0,97 & 1,82 & 3,6 & 630 & 0,776 & 64,4 & 83,0 & 3,3 & & \\
\hline & \#2 & 1,770 & 1,6 & 0,179 & 1,8 & 32 & 0,382 & 2,4 & 69 & 2,14 & 0,58 & 3,6 & 104 & 0,745 & 53,3 & 71,6 & 3,1 & & \\
\hline & \#3 & 2,823 & 1,6 & 0,319 & 1,8 & 69 & 0,064 & 2,4 & 14 & 0,20 & 1,26 & 3,6 & 271 & 0,773 & 67,5 & 87,3 & 3,6 & 80,6 & 4,7 \\
\hline \multirow[t]{3}{*}{ V-252 } & $\# 1$ & 2,036 & 1,7 & 0,221 & 1,8 & 50 & 0,102 & 2,4 & 23 & 0,46 & 1,52 & 3,6 & 345 & 0,732 & 65,2 & 89,0 & 4,1 & & \\
\hline & \#2 & 0,119 & 2,4 & 0,014 & 8,5 & 3 & 0,032 & 2,5 & 8 & 2,33 & 0,85 & 3,6 & 209 & 0,753 & 34,6 & 45,9 & 2,8 & & \\
\hline & $\# 3$ & 2,117 & 1,6 & 0,330 & 1,8 & 68 & 0,049 & 2,5 & 10 & 0,15 & 1,36 & 3,6 & 280 & 0,798 & 49,5 & 62,0 & 2,4 & 65,6 & 12,6 \\
\hline \multirow[t]{3}{*}{ V-266 } & $\# 1$ & 0,099 & 2,3 & 0,020 & 4,1 & 13 & 0,045 & 2,5 & 30 & 2,21 & 0,40 & 2,4 & 260 & 0,553 & 23,8 & 43,0 & 3,2 & & \\
\hline & \#2 & 0,022 & 4,5 & 0,004 & 75,2 & 12 & 0,013 & 3,6 & 43 & 3,68 & 0,02 & 2,4 & 69 & 0,360 & 26,7 & 74,3 & 29,1 & & \\
\hline & $\# 3$ & 0,198 & 1,6 & 0,036 & 2,4 & 42 & 0,041 & 2,5 & 49 & 1,16 & 0,12 & 3,4 & 143 & 0,582 & 35,4 & 60,7 & 4,1 & 59,3 & 9,1 \\
\hline
\end{tabular}

a: Amount of helium is given in nano-cubic-cm in standard temperature and pressure.

${ }^{b}$ : Amount of radioactive elements are given in nanograms.

c: Ejection correct. (Ft): correction factor for alpha-ejection (according to Farley et al., 1996).

d: Uncertainty of the sample average age is 1 standard error, as $(\mathrm{SD}) /(n)^{1 / 2}$; where $\mathrm{SD}=$ standard deviation of the age replicates and $n=$ number of age determinations. 